\title{
Prevalencia de Obesidad en escolares
}

\author{
Josefa Plaza Almeidaa , José Manuel Siurana Rodríguez ${ }^{\mathrm{b}}$, Llanos Vergara González ${ }^{\mathrm{c}}$, \\ Fernando Mateos Rodríguez ${ }^{d}$, María Isabel Romero Belluga ${ }^{e}$
}

a Pediatra de Atención Primaria. Centro de Salud Zona 1 de Albacete.

${ }^{b}$ Médico Interno Residente de Pediatría. Complejo Hospitalario Universitario de Albacete.

c Enfermera de Atención Primaria. Centro de Salud Zona 1 de Albacete.

d Medicina Interna. Complejo Hospitalario Universitario de Albacete.

e Médico Interno Residente de Medicina de Familia. Complejo Hospitalario Universitario de Albacete.

Correspondencia: Josefa Plaza Almeida. Avda. Estación $n^{\circ} 6,9^{\circ} \mathrm{D}$, 02001 - Albacete. Telf.: 967247565, e-mail: plazaalmeida@hotmail.com.

Recibido el 10 de enero de 2008.

Aceptado para su publicación el 29 de enero de 2008.

\section{RESUMEN}

Objetivos. Conocer la prevalencia de obesidad en los niños de 6 a 8 años. Valorar si la asistencia al comedor escolar y las vacaciones del periodo estival influyen de manera significativa en la obesidad de los niños.

Diseño. Estudio observacional longitudinal.

Emplazamiento. Un colegio público urbano de la Zona Básica de Salud número 1 de Albacete.

Participantes. 102 niños de primero y segundo cursos de educación primaria.

Mediciones principales. Las medidas antropométricas peso, talla e índice de masa corporal (IMC) fueron evaluadas en los sujetos de estudio durante el mes de mayo de 2007. De nuevo, en septiembre, se obtuvieron estos datos somatométricos en los mismos niños, así como la medición del perímetro abdominal en los que resultaron obesos. Para el cálculo de los percentiles se utilizaron las tablas de peso, talla, IMC y perímetro abdominal según edad y sexo de la fundación Orbegozo.

Resultados. La prevalencia de obesidad en la primera evaluación fue del $23,5 \%$ y la de sobrepeso del $33,3 \%$. En la segunda medición los valores ascendieron a 28,2 y $36,4 \%$ respectivamente. Comparando el valor medio del IMC antes y después del verano, se observó una diferencia estadísticamente significativa, siendo superior después del verano (18,3 vs 18,7 Kg/m2; $p=0,005)$.

Conclusiones. Encontramos una alta prevalencia de obesidad en nuestros escolares, sin relación con la asistencia al comedor escolar, sin embargo, tras el periodo estival, se comprobó un aumento estadísticamente significativo en el IMC.

Palabras clave. Obesidad. Infancia.

\section{ABSTRACT}

\section{Prevalence of obesity in school children.}

Objectives. To determine the prevalence of obesity in children from 6 to 8 years old. To determine if school dinners and the holiday period significantly affect obesity in children.

Design. Longitudinal, observational study.

Setting. A state school in an urban area in Basic Health Area number 1 in Albacete.

Participants. 102 children in the first and second year of primary school.

Main measurements. The anthropometric measurements of weight, height and body mass index (BMI) of the subjects were taken during May 2007. In September these measurements were taken again and the abdominal perimeter was also measured in subjects who were obese. The percentiles were calculated using the Fundación Orbegozo charts for weight, height, BMI and abdominal perimeter according to age and gender.

Results. The prevalence of obesity in the first evaluation was $23.5 \%$ and the prevalence of being overweight was $33.3 \%$. In the second evaluation these values increased to $28.1 \%$ and $36.4 \%$, respectively. There was a statistically significant difference in the BMI index before and after the summer holidays, the BMI being higher after the summer holidays (18.3 vs $18.7 \mathrm{Kg} / \mathrm{m} 2 ; \mathrm{p}=0.005$ ).

Conclusions. We found a high prevalence of obesity in our school children. Obesity was not related to school dinners. However, after the summer holidays there was a significantly increase in BMI.

Key Words. Obesity Childhood. 


\section{INTRODUCCIÓN}

La Organización Mundial de la Salud (OMS) considera la obesidad como una epidemia de distribución mundial ${ }^{1}$. Comparando con otros países, España se encuentra en una posición intermedia en el porcentaje de adultos obesos $(14,5 \%$ según los resultados del estudio SEEDO 2000²), sin embargo, la población infantil española presenta una de las cifras más altas.

La obesidad infantil se ha triplicado en los últimos 15 años. El pico máximo de obesidad en la infancia se encuentra entre los 6 y 12 años, edad en la que el $16,1 \%$ de los niños son obesos ${ }^{3}$ (Enkid, 2000). Se calcula que en los países desarrollados los costes directos e indirectos que ocasionan el sobrepeso y la obesidad alcanzan un $7 \%$ del gasto sanitario total ${ }^{4}$. Los grupos sociales menos favorecidos en renta y cultura sufren más este problema de salud ${ }^{2}$.

La obesidad tiene múltiples factores etiológicos, los más importantes se relacionan con el estilo de vida actual. Se han modificado los hábitos alimentarios, sustituyéndose la "dieta mediterránea" por comidas más fáciles de preparar y consumir pero que aportan más energía. Por otra parte, ha aumentado el sedentarismo, sustituyéndose la actividad física y el deporte por el entretenimiento pasivo (televisión, ordenadores y consolas de videojuegos).

Los objetivos de nuestro estudio son: en primer lugar, conocer la prevalencia de obesidad en los escolares de nuestra zona de salud; en segundo lugar, comprobar si la asistencia al comedor escolar supone un factor protector o de riesgo para la obesidad en nuestros escolares; en tercer lugar, comprobar si las vacaciones estivales contribuyen a reducir o empeorar este problema de salud.

\section{MATERIAL Y MÉTODO}

Se trata de un estudio observacional longitudinal en el que han sido estudiados los alumnos del Colegio Virgen de los Llanos de Albacete en los cursos de $1^{\circ}$ y $2^{\circ}$ de primaria. Es un colegio público de 3 líneas que atiende a una población de nivel socioeconómico medio-bajo, con un porcentaje de niños inmigrantes del $10 \%$. Los datos somatométricos se obtuvieron, en los mismos sujetos, en dos ocasiones diferentes: mayo y septiembre de 2007. En la primera medición formó parte del estudio un total de 102 niños de edad comprendida entre 6 y 7 años: 48 varones (47\%) y 54 mujeres (53\%), mientras que en la segunda acudieron $85(83,3 \%): 40$ varones (47\%) y 45 mujeres (53\%). El peso y la talla fueron medidos, respectivamente, mediante báscula y tallímetro homologados. Posteriormente se calculó el índice de masa corporal $(\mathrm{Kg} / \mathrm{m} 2)$. La medida de la circunferencia abdominal se realizó en el punto medio entre el reborde costal inferior de la última costilla y las crestas ilíacas. Para el cálculo de los percentiles se utilizaron las tablas de peso, talla, IMC y perímetro abdominal, según edad y sexo, de la fundación Orbegozo. Se consideró obesidad un IMC igual o superior al percentil 95 para la edad y el sexo, y sobrepeso un IMC igual o superior al percentil 85 para la edad y el sexo.

Para los cálculos estadísticos se utilizó el programa SPSS versión 12.0. Se realizó un análisis descriptivo de la muestra de pacientes expresado mediante la media y la desviación estándar. En el análisis bivariado, para la comparación de subgrupos en cuanto a variables continuas se utilizó la t de Student para muestras independientes y relacionadas. Se consideró que las diferencias eran estadísticamente significativas para una $p<0,05$.

\begin{tabular}{|c|c|c|c|}
\hline Primera evaluación & Total & Niños ( $n=48)$ & Niñas $(n=54)$ \\
\hline Edad (años) & $6,55 \pm 0,5$ & $6,56 \pm 0,5$ & $6,54 \pm 0,5$ \\
\hline Peso (kg) & $29,20 \pm 7,51$ & $28,67 \pm 6,98$ & $29,67 \pm 8$ \\
\hline Talla (cm) & $125,20 \pm 13,32$ & $126,65 \pm 6,66$ & $123,92 \pm 17,19$ \\
\hline IMC (Kg/m2) & $18,07 \pm 3,27$ & $17,71 \pm 3,24$ & $18,40 \pm 3,29$ \\
\hline Percentil IMC (\% mayor) & 26,5 en P25-50 & 25 en P25-50 & 27,8 en $\mathrm{P} 25-50$ y 27,8 en $\mathrm{P} 50-75$ \\
\hline$P>95$ de IMC & $23,5 \%$ & $20,8 \%$ & $25,9 \%$ \\
\hline $\mathrm{P}>85$ de IMC & $33,3 \%$ & $31,2 \%$ & $35,1 \%$ \\
\hline Van a comedor (\%) & 30,4 & 25 & 35,2 \\
\hline Segunda evaluación & Total & Niños $(n=40)$ & Niñas $(n=45)$ \\
\hline Peso (kg) & $31,60 \pm 8,62$ & $30,72 \pm 8,25$ & $32,39 \pm 8,96$ \\
\hline Talla (cm) & $129,08 \pm 7,05$ & $128,83 \pm 6,90$ & $129,31 \pm 7,26$ \\
\hline IMC (Kg/m2) & $18,75 \pm 3,84$ & $18,31 \pm 3,88$ & $19,10 \pm 3,76$ \\
\hline Percentil IMC (\% mayor) & 20,6 en P25-50 & 18,8 en $P>97$ & 25,9 en P25-50 \\
\hline Perímetro abdominal $(n=29)$ & $71,32 \pm 6,45$ & $71,54 \pm 7,37$ & $71,17 \pm 5,96$ \\
\hline Percentil PA (\% mayor) & 10,8 en $P>97$ & 8,3 en $P>97$ & 13 en $P>97$ \\
\hline $\mathrm{P}>95$ de IMC & $28,2 \%$ & $27,5 \%$ & $28,8 \%$ \\
\hline $\mathrm{P}>85$ de IMC & $36,4 \%$ & $35 \%$ & $40 \%$ \\
\hline
\end{tabular}

Tabla 1. Características de la población. 


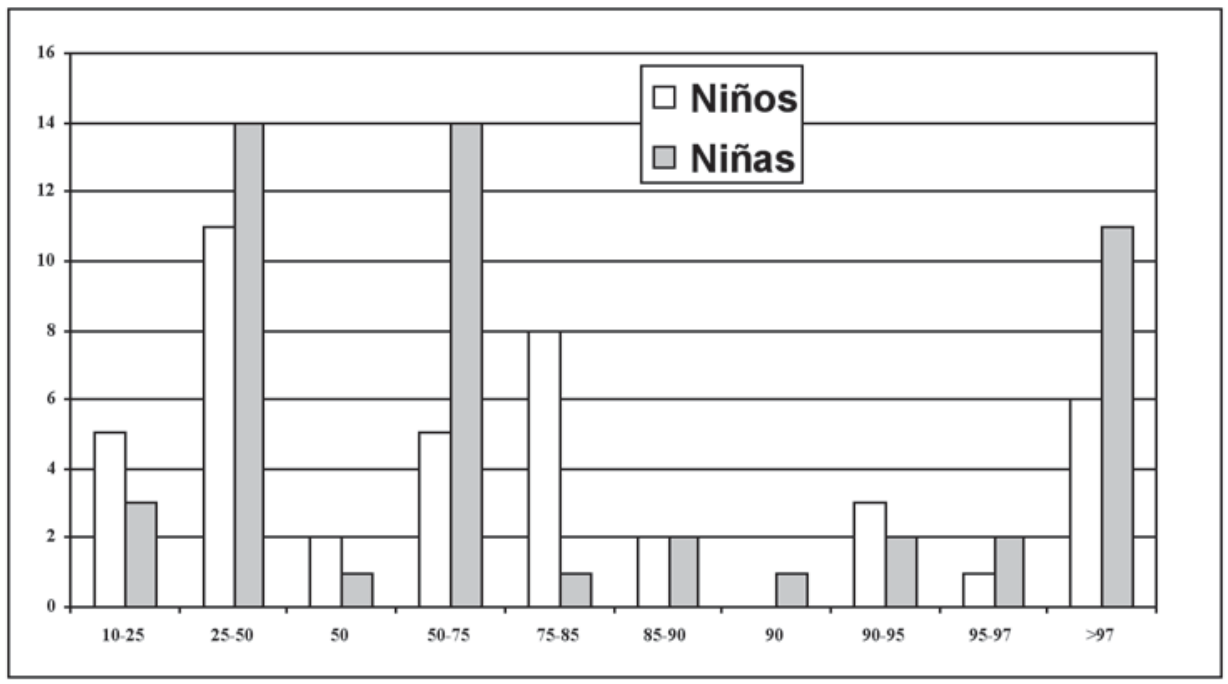

Figura 1. Prevalencia de cada uno de los percentiles de IMC en la primera evaluación.

\begin{tabular}{lccc}
\hline & Asistencia al comedor & No asistencia al comedor & $\mathbf{p}$ \\
\hline Peso $(\mathrm{kg})$ & $27,27 \pm 7,02$ & $30,05 \pm 7,61$ & $\mathrm{NS}$ \\
Talla $(\mathrm{cm})$ & $121,40 \pm 21,24$ & $126,86 \pm 7,32$ & $\mathrm{NS}$ \\
IMC $(\mathrm{Kg} / \mathrm{m} 2)$ & $17,28 \pm 3,5$ & $18,42 \pm 3,12$ & $\mathrm{NS}$ \\
\hline
\end{tabular}

Tabla 2. Mediciones de los parámetros somatométricos según asistencia o no al comedor escolar. NS: diferencia estadísticamente no significativa.

\begin{tabular}{lccc}
\hline & Antes del verano & Después del verano & p \\
\hline Peso $(\mathrm{kg})$ & $29,94 \pm 7,86$ & $31,60 \pm 8,62$ & 0,000 \\
Talla $(\mathrm{cm})$ & $125,55 \pm 14,19$ & $129,08 \pm 7,05$ & 0,009 \\
IMC $(\mathrm{Kg} / \mathrm{m} 2)$ & $18,37 \pm 3,49$ & $18,73 \pm 3,82$ & 0,005 \\
\hline
\end{tabular}

Tabla 3. Medidas de los datos somatométricos antes y después del periodo vacacional.

\section{RESULTADOS}

Las características de los escolares estudiados se muestran en la tabla 1. Aunque en la primera determinación el percentil de IMC más prevalente fue el 25$50(26,5 \%)$, la proporción de obesos $(P \geq 95)$ alcanzó el $23,5 \%$ y la de sujetos con sobrepeso $(P \geq 85)$ el $33,3 \%$. La distribución de la población según su percentil y su distribución por género se puede ver en las figura 1. No se hallaron diferencias estadísticamente significativas entre sexos para las variables peso, talla e IMC.

El porcentaje de obesos en la segunda determinación fue del $28,2 \%$ y el de niños con sobrepeso del $36,4 \%$. El percentil de mayor prevalencia fue también el 25-50 $(20,6 \%)$. No se hallaron tampoco en esta ocasión diferencias estadísticamente significativas entre sexos para las variables peso, talla e IMC.

Del total de los sujetos estudiados, asistía al comedor escolar el $30,4 \%$ (25\% de los niños y $35,2 \%$ de las niñas). Comparando los datos somatométricos de los sujetos que asistían al comedor con los de los que no lo hacían (tabla 2), encontramos que no existían diferencias estadísticamente significativas en el peso (27,2 vs $30,0 \mathrm{Kg})$, la talla (121,4 vs $126,8 \mathrm{~cm}$ ) o el IMC $\left(17,2\right.$ vs $\left.18,4 \mathrm{Kg} / \mathrm{m}^{2}\right)$.

Para evaluar la influencia del periodo vacacional en los 85 sujetos que pudieron ser evaluados en las dos ocasiones (tabla 3), se comparó el valor medio del peso, la talla y el IMC antes y después del verano. Se pudo apreciar que la diferencia era estadísticamente significativa $(p<0,05)$ en las tres variables, siendo todas las medias superiores después del verano: peso $(29,9$ vs $31,6 \mathrm{Kg})$, talla $(125,5$ vs $129,0 \mathrm{~cm})$ e IMC $(18,3$ vs $\left.18,7 \mathrm{Kg} / \mathrm{m}^{2}\right)$.

\section{DISCUSIÓN}

La prevalencia de obesidad encontrada en nuestros escolares es alta $(23.5 \%)$ en relación al estudio En- 
kid de 1998-2000, realizado por regiones y con una media española de 13,9\%. Esta diferencia se puede justificar por varios motivos: en primer lugar, nuestra comunidad autónoma, encuadrada en el Sureste de España, se encuentra entre las de mayor prevalencia junto a Andalucía, Murcia y Canarias (15-18\%); en segundo lugar, en el estudio Enkid se ha utilizado como criterio para definir obesidad el percentil 97 de las tablas de Orbegozo, mientras que nosotros hemos incluido a los niños con IMC mayor o igual al Percentil 95 de esas mismas tablas, apoyándonos en las recomendaciones del Comité de Nutrición de la Asociación Española de Pediatría ${ }^{5}$; en tercer lugar, hay que tener en cuenta el incremento anual de este problema de salud y comparar las fechas en que se realizaron las mediciones, en este caso 7 años más tarde (1998-2000 en el estudio Enkid frente a 2007 en el nuestro). Se ha calculado un incremento anual del $\mathrm{IMC}^{6}$ de $0,5 \mathrm{Kg} / \mathrm{m}^{2}$.

Otros estudios más recientes, como los realizados por Albañil et al en Fuenlabrada o Leis et al en Galicia, aportan, mediante mediciones realizadas en 2003, cifras de prevalencia de obesidad a los 6 años del $12,4 \%^{7}$ y del $14,4 \%{ }^{8}$, respectivamente. Dichos resultados no son comparables con los nuestros, ya que utilizan tablas de los Centers for Diseases Control and Prevention (CDC) de 20009. El estudio "Cuatro provincias" (Madrid, Orense, Murcia y Cádiz) obtuvo prevalencias del 8,5-15,7\% en niños de 6-7 años ${ }^{10}$.

En nuestra Comunidad Autónoma contamos con el "Estudio de Cuenca"11, que presentó cifras del 3,9\% para obesidad y $26,16 \%$ para sobrepeso, muy inferiores a las observadas en el resto de los estudios españoles. Cuatro años más tarde, una nueva edición del "Estudio de Cuenca" obtuvo cifras muy superiores de obesidad $(8.8 \%)^{12}$. Este estudio utilizó gráficas internacionales con los Criterios de la International Obesity Task Force ${ }^{13}$. Respecto a estás gráficas, se ha referido una menor sensibilidad para detectar cifras de obesidad, sobre todo en el sexo masculino ${ }^{14}$. Estas mismas tablas, elaboradas por Cole y publicadas en el año $2000^{13}$, se han utilizado en la Encuesta de Salud de Castilla-La Mancha (CLM), realizada en el $2^{\circ}$ semestre del año 2006 por el área del Observatorio de Salud de la Fundación para la Investigación Sanitaria en CLM $(\text { FISCAM })^{15}$. En la población entre 2 y 15 años se obtuvieron unas cifras de exceso de peso del 29,2\%; además, son los niños de menor edad los de mayor prevalencia, de tal manera que entre los 2 y 5 años presenta exceso de peso el $35 \%$ de los casos (sobrepeso el $15 \%$ y obesidad el 19,4\%). Son las clases socioeconómicas más favorecidas las que presentan menor prevalencia de exceso de peso (23\%) frente a las menos favorecidas (29\%), siendo éstas las cifras que más se asemejan a las nuestras.

Nuestros datos coinciden también con otro estudio realizado en niños de 11 años, seleccionados por su pertenencia a una consulta de pediatría de la misma Zona de Salud durante el año 2007, donde, utilizando las mismas tablas y criterios diagnósticos, se observó una prevalencia de obesidad del $23,20 \%{ }^{16}$.

Dada la polémica que ha suscitado la calidad de las comidas en los comedores escolares a nivel de medios de comunicación, quisimos valorar si la asistencia a estos constituye una variable asociada al sobrepeso. No observamos diferencias estadísticamente significativas en la prevalencia de obesidad entre los niños que asisten o no al comedor escolar.

En cuanto a la influencia del verano en los datos de la somatometría de estos niños, encontramos un aumento del IMC al finalizar dicho periodo estival. La segunda medición no pudo ser llevada a cabo en el total de los escolares, pudiendo existir algún sesgo de selección en esta ocasión, sin embargo, aunque durante el verano los niños realizan más ejercicio físico, la diferencia podría ser debida a que también aumenta el apetito y la ingesta de alimentos no recomendables en horarios irregulares.

La medida de la circunferencia abdominal en los escolares que resultaron obesos aportó cifras por encima del percentil 75 para la edad y el sexo, punto de corte propuesto como criterio de Síndrome metabólico ${ }^{5}$. Coincidimos con publicaciones recientes ${ }^{17}$ en que, como casi todos los obesos presentaron un perímetro de la circunferencia abdominal superior al percentil 90, tal vez no sea aconsejable utilizar dicho parámetro como un componente del síndrome metabólico, resultando más fácil usar el IMC.

Nuestro estudio presenta limitaciones, ya que el número de casos es pequeño y no permite extrapolar los datos obtenidos. Tiene la ventaja de aportar datos sobre nuestra población, con el fin de conocer la situación concreta, paso previo a la intervención sanitaria preventiva ${ }^{18}$. Los niños pequeños y las familias deben ser los principales grupos diana en las estrategias de prevención ${ }^{19}$. Creemos que las revisiones de salud del niño sano realizadas en Atención Primaria, junto con la educación para la salud en las escuelas, son lugares adecuados para la prevención, captación y seguimiento de este importante problema de salud, dada la dificultad para realizar un tratamiento eficaz.

\section{AGRADECIMIENTOS}

A Manuel Jesús García Cabello, Director del Colegio Virgen de los Llanos por su inestimable colaboración en este estudio. A Jesús López-Torres, por la colaboración en la redacción del manuscrito.

\section{BIBLIOGRAFÍA}

1. Organización Mundial de la Salud (OMS). La obesidad y la gestión de la prevención de la epidemia mundial. Serie de Informes Técnicos 894. Ginebra: OMS; 2000.

2. Aranceta Bartrina J, Pérez Rodrigo C, Serra Majem L, Ribas Barba L, Quiles Izquierdo J, Vioque J, et al. Prevalencia de la obesidad en España: resultado del estudio SEEDO 2000. Med Clin (Barc) 2003; 120(16):608-12. 
3. Serra Majem L, Ribas Barba L, Aranceta Bartrina J, Pérez Rodrigo C, Saavedra Santana P. Epidemiología de la obesidad infantil y juvenil en España. Resultados del Estudio enKid (1998-2000). En: Serra Majem L, Aranceta Bartrina J, editores. Obesidad infantil y juvenil. Estudio enKid. Vol.2. Barcelona: Masson S.A.; 2001. p. 81-108.

4. Instituto Bernard Krieff. Informe: estudio de costes sociales y económicos de la obesidad y sus patologías asociadas. Madrid: Instituto Bernard Krieff; 1999.

5. Dalmau Serra J, Alonso Franch M, Gómez López L, Martínez Costa C, Sierra Salinas C. Obesidad infantil. Recomendaciones del Comité de Nutrición de la Asociación Española de Pediatría. Parte II. Diagnóstico. Comorbilidades. Tratamiento. An Pediatr (Barc) 2007; 66:294-304.

6. Kavey RE, Daniela SR, Lauer RM, Atkins DL, Hayman LL, Taubert K. American Heart Association guidelines for primary prevention of atherosclerotic cardiovascular disease beginning in childhood. J Pediatr 2003; 142:368-72.

7. Albañil Ballesteros MR, Sánchez Martín M, de la Torre Verdú M, Olivas Domínguez A, Sánchez Méndez MY, Sanz Cuesta T. Prevalencia de obesidad a los 14 años en cuatro consultas de atención primaria. Evolución desde los dos años. An Pediatr (Barc) 2005; 63:39-44

8. Leis R, Martinez A, Novo A, Tojo R. Trend of obesity prevalence in children from Galicia (NW of Spain) 1979-2001. GALINUT Study. J Pediatr Gastroenterol Nutr 2003; 36:553.

9. Grummer-Strawn LM, Garza C, Johnson CL. Childhood growth charts. Pediatrics 2002; 109:141-147.

10. Rodríguez-Artalejo F, Garcés C, Gorgojo L, López E, Martín-Moreno JM, Benavente $\mathrm{M}$, et al. Dietary patterns among children aged 6-7 in four Spanish cities widely differing cardiovascular mortality. Eur J Clin Nutr 2002; 56:141-8.

11. Martínez F, Salcedo F, Rodríguez F, Martínez V, Domínguez LM, Torrijos R. Prevalencia de la obesidad y mantenimiento del estado ponderal tras un seguimiento de 6 años en niños y adolescentes: estudio de Cuenca. Med Clin (Barc) 2002; 119: 327-30.

12. Morant Sánchez A, Solera Martínez M, Martínez Vizcaíno V, Salcedo Aguilar F, Franquelo R, Rodríguez Artalejo F, et al. Prevalencia de obesidad y tendencia de los factores de riesgo cardiovascular en escolares de 1992 a 2004: estudio de Cuenca. Med Clin (Barc) 2006; 126:681-5.

13. Cole TJ, Bellizzi MC, Flegal KM, Dietz WH. Establishing a Standard definition for child overweight and obesity worldwide: international survey. BMJ 2000; 320:1240-3.

14. Reilly JJ, Wilson ML, Summerbell CD, Wilson DC. Obesity: Diagnosis, prevention, and treatment; evidence based answer to common questions. Arch Dis Child 2002; 86:392-4.

15. Caballero A, Domper J, García MA, Hidalgo A, Modesto RM, Morillo F, et al. Encuesta de Salud de Castilla La Mancha 2006. (Monografía en CD-ROM).

16. Plaza Almeida J, López Sánchez FJ, Sánchez López C, Vergara González LI, García Segura M, Arenas García G. Detección y seguimiento de niños obesos de 11 años. $1^{\text {a }}$ Reunión de la Asociación de Pediatría de Atención Primaria de Castilla-La Mancha 2007.

17. Tapia Ceballos L, López Siguero JP, Jurado Ortiz A. Prevalencia del síndrome metabólico y sus componentes en niños y adolescentes con obesidad. An Pediatr (Barc) 2007; 67:352-61.

18. Carraro R, García Cebrián M. El papel de la prevención en la contención de la epidemia de obesidad. Eur J Clin Nutr 2003: 57(Suppl 1):S94-S96.

19. Aranceta J, Pérez-Rodrigo C, Serra Majem L, Bellido D, de la Torre ML, Formiguera $X$, et al. Prevention of overweight and obesity: a Spanish approach. Public Health Nutr 2007; 10:118793. 